\title{
Levantamento manual de carga repetitivo de curta duração com e sem o uso de cinto pélvico: efeito sobre a atividade EMG normalizada por diferentes indicadores
}

\section{Effect of a pelvic belt on EMG activity during manual load lifting}

\author{
Mauro Gonçalves \\ Marcelo Pinto Pereira
}

1 Universidade Estadual Paulista Júlio de Mesquita Filho. Instituto de Biociências. Departamento de Educação Física. Programa de Pós-graduação em Ciências da Motricidade Humana. Campus Rio Claro, SP.

Recebido em 06/05/08 Revisado em 10/09/08 Aprovado em 13/12/08
Resumo - A capacidade de levantamento e abaixamento manual de carga (LAMC) continua preocupando as indústrias e o uso da eletromiografia (EMG) se apresenta como uma alternativa no estudo da musculatura envolvida nessa atividade. Entretanto, a reprodutibilidade destas medidas torna-se primordial. Assim, esse estudo teve como objetivo principal analisar a influência do uso do cinto pélvico sobre a atividade EMG dos músculos eretores da espinha (ER) e reto femoral (RF), durante LAMC e durante a contração isométrica voluntária máxima (CIVM) de extensão do tronco, realizada antes (PRÉ) e após essa atividade, assim como analisar a variabilidade (CV) do sinal EMG quando normalizadas por três métodos: pelo pico (PICO) e pela média (MED) da atividade EMG e pelo sinal obtido durante a CIVM. Para isso, 8 voluntários realizaram LAMC de $15 \%$ e $25 \%$ peso corporal (PC), com e sem o uso do cinto pélvico por $1 \mathrm{~min}$. Obtiveram-se os valores do Coeficiente da Variação (CV) do sinal EMG do ER e RF durante os LAMCs. Durante as CIMVs, obtiveram-se os valores de tração na célula de carga e as variáveis eletromigráficas RMS, Frequência Média, Frequência Mediana e Potência Total do músculo ER. Os resultados demonstram menores valores de CV quando o sinal EMG foi obtido pelo PICO, sendo, portanto, esse método preferível por apresentar menor variabilidade. Durante as CIVMs, somente a força de tração na célula de carga se apresentou menor após o LAMC de 25\%PC sem o uso do cinto pélvico $(\mathrm{p}=0,035)$, sugerindo rápida recuperação dos ER após o LAMC por 1 minuto.

Palavras-chave: Eletromiografia; Fadiga; Levantamento manual.

Abstract - Manual lifting (ML) capacity is still a matter of concern for industry administrators and electromyography (EMG) seems to be a good alternative for the evaluation of muscles involved in this task. However, the reliability of these measures is very important. Thus, the objective of this study was to evaluate the influence of a pelvic belt on EMG activity of the erector spinus (ES) and rectus femoralis (RF) muscles during ML and during maximal voluntary contractions (MVC) of trunk extension performed before (baseline) and after ML. In addition, the variability in the EMG signal normalized by the following three different methods was evaluated: peak EMG activity, mean EMG activity, and EMG activity obtained during MVC. Eight volunteers performed ML of $15 \%$ and $25 \%$ of their body weight for 1 minute in the presence or absence of a pelvic belt. The coefficient of variation (CV) of the EMG signal obtained for the ES and RF muscles was calculated during ML. Load cell traction values and the electromyographic variables RMS, median frequency, mean power frequency and total power of the ES muscle were obtained during MVC. The results showed lower CV (smaller variability) when the EMG signal was normalized by peak activity, with this method thus being preferable. During MVC, only the load cell traction value differed from baseline after ML of $25 \%$ body weight without the pelvic belt ( $p=0.035)$, a finding suggesting rapid recovery of ES muscle after ML for 1 minute.

Key words: Electromyography; Fatigue; Manual lifting. 


\section{INTRODUÇÃO}

A capacidade de levantamento manual de carga pelo homem continua preocupando as indústrias dos mais diversos setores de produção, devido ao número expressivo de ocorrência de dor lombar, proveniente do número excessivo de levantamentos de carga, se realizado de forma incorreta, ocasionando sobrecargas nos tecidos não contrateis como fascias e ligamentos, podendo, inclusive, ocorrer como resultado, uma herniação posterior do núcleo pulposo1. Esta capacidade está limitada a diversos fatores como: a força do trabalhador, a máxima compressão discal possível sem que haja risco de lesões (sendo que posturas cifóticas causam maior força de cisalhamentol), a capacidade cardiovascular (logo que diferentes técnicas de levantamento manual de carga trazem diferentes respostas a variáveis como consumo de oxigênio e freqüência cardíaca ${ }^{2}$ ) e a percepção do desconforto ao levantamento. Muitos são os fatores estudados sobre o levantamento de carga que podem influenciar na sua eficiência, inclusive, como causas de muitas das lesões ocasionadas por este movimento, alguns destes fatores são: a postura em que as articulações estão no início e durante o levantamento, a quantidade de carga, a velocidade de execução, a altura em que a carga se encontra no início do levantamento, os vários tipos e a presença ou não de puxadores, o uso de acessórios como o cinto de suporte lombar, a pressão intra-abdominal, a fadiga muscular e outros ${ }^{3}$.

A quantidade de carga é um fator importante para se realizar um levantamento manual seguro, e foi correlacionada com outras variáveis como a linearidade com a pressão intra-abdominal ${ }^{4}$ e o aumento da força ao nível lombar (L4-L5)5. Para avaliar a ação dos músculos durante o levantamento manual de carga, muitos estudos utilizaram como instrumento a eletromiografia, permitindo, assim, uma correlação entre o esforço muscular e as forças externas que atuam no ser humano durante esta atividade, com evidência para os músculos da coluna lombar, quadril, isquiotibiais e os músculos abdominais ${ }^{6}$ que estão correlacionados com a quantidade de peso levantada, a postura do tronco durante o levantamento e a distância entre a carga e o centro do corpo, como o momento externo, o peso levantado, a postura corporal, a localização do centro de gravidade do peso e a velocidade de levantamento.

Outro mecanismo utilizado com intuito de proteger a coluna lombar de lesões pelo esforço durante o levantamento de carga é o uso de cinto pélvico. Entretanto, as opiniões continuam controversas quanto a esse tema. Esta prática parece pouco difundida nas situações de trabalho ou de atividade de vida diária, sendo rotina para aqueles que treinam o levantamento de peso olímpico e não olímpico.

O fenômeno da fadiga tem sido estudado pela progressiva e contínua atividade voluntária que leva a um aumento da amplitude e duração dos potenciais de ação devido aos processos de recrutamento por somação das unidades motoras? Destacam-se, então, duas propriedades principais do sinal eletromiográfico (EMG) que podem afetar o espectro de freqüência: o comportamento de disparo das unidades motoras (UM) e a forma do potencial de ação das unidades motoras (PAUM). Com isto, outra possibilidade na identificação da fadiga eletromiográfica é a modificação espectral, que pode ser monitorada e quantificada seguindo alguns indicadores característicos do espectro de freqüência, como a freqüência média, mediana e área total do espectro ${ }^{8,9}$.

Em contrações isométricas, pode haver um consenso em existir um aumento da resposta eletromiográfica à medida que aumenta a tensão muscular ${ }^{10}$, porém dependente da porcentagem da carga utilizada em relação à Contração Isométrica Voluntária Máxima (CIVM) ${ }^{7}$.

Associado às diversas variáveis que influenciam a interpretação do registro EMG está o método de normalização. Com intuito de identificar uma provável padronização do sinal eletromiográfico que represente um comportamento reprodutível de um músculo durante um movimento específico, a normalização apresenta-se como uma ferramenta que permite assim comparar diferentes voluntários quando submetidos à mesma tarefa. Este procedimento tem sido utilizado em biomecânica por diversos autores ${ }^{11}$ e particularmente para avaliar a reprodutibilidade do sinal eletromiográfico. Destaque aos estudos de Winter ${ }^{11,12}$ que apresentam, como no presente estudo, três formas de normalização: pela contração voluntária máxima, pelo pico ${ }^{9} \mathrm{e}$ pela média do sinal ${ }^{12}$.

Outro fator importante na análise EMG é a reprodutibilidade dos resultados intersujeitos, normalmente analisada pelo coeficiente de variação (CV), sendo que menores valores representam maior reprodutibilidade ${ }^{13}$. O CV reflete a dispersão dos dados ao redor da média e é calculado pela raiz quadrada do desvio padrão sobre a média. Os valores altos ou baixos não são considerados bons 


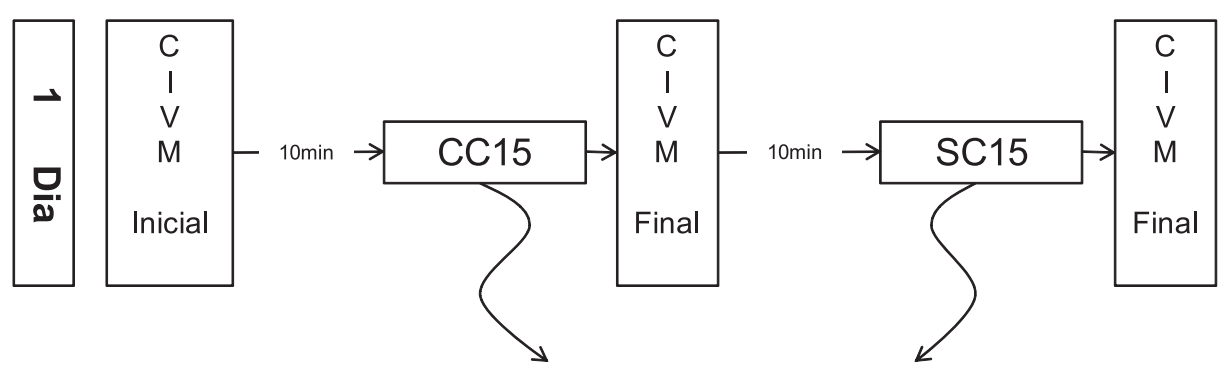

Ordem randômica

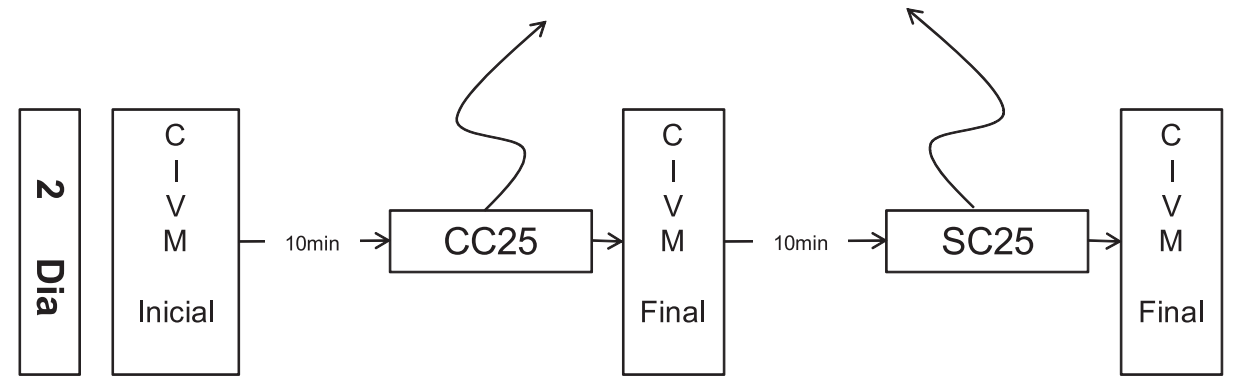

Figura 1: Protocolo de coleta realizado nos dois dias de teste: CIVM: contração isométrica voluntária máxima; CC - uso do cinto pélvico; SC - sem uso do cinto pélvico; 15 - uso de 15\% peso corporal como carga; 25 - uso de 25\% peso corporal como carga.

ou ruins. Algum grau de variabilidade é necessário para demonstrar a reprodutibilidade. Um CV baixo sugere homogeinedade do grupo, o que permite criar um diagnóstico ou modelo que pode ser comparado a outras avaliações realizadas. Esta é a base na qual a média dinâmica da eletromiografia tem sido considerada como valor de normalização quando estudando eventos dinâmicos.

Por outro lado, uma vez que a análise eletromiográfica de contrações isométricas têm sido uma alternativa para verificar a capacidade de resistência dos músculos da coluna lombar em função do tempo de contração ${ }^{8,14}$, a reprodutibilidade destas medidas torna-se primordial.

Pelo exposto, o presente estudo teve como objetivo analisar a influência do uso do cinto pélvico sobre a atividade dos músculos eretores da espinha e reto femoral durante o levantamento e abaixamento manual de diferentes percentuais de cargas, assim como analisar a variabilidade (CV) desta atividade quando normalizadas por três diferentes métodos.

\section{PROCEDIMENTOS METODOLÓGICOS}

O presente estudo foi realizado por 8 voluntários homens, com idade de 20,7 $\pm 2,1$ anos, altura de $1,72 \pm 0,03 \mathrm{~m}$ e massa corporal de $65,28 \pm 8,95 \mathrm{Kg}$, sem antecedentes de doenças músculo-esqueléticas.

No período de 24 hs prévio ao da primeira coleta, os voluntários estiveram no laboratório para familiarização com os procedimentos a serem realizados durante os dias seguintes. O presente estudo foi aprovado pelo Conselho de Ética local (Parecer número 6329) e os voluntários assinaram Termo de consentimento livre e esclarecido sobre todas as tarefas que iriam ser realizadas nos momentos de coleta.

\section{Procedimentos}

O protocolo foi realizado em dois dias diferentes (intervalo mínimo de 24 horas e de no máximo 96 horas entre eles), sendo realizadas duas atividades por dia (distantes entre si em 10 minutos) de forma aleatória, somando, portanto, quatro diferentes protocolos: levantamento de duas cargas (15\% e $25 \%$ do peso corporal), com o uso do cinto pélvico (CC) e sem sua utilização (SC).

Os voluntários realizaram em cada dia de teste, antes dos levantamentos e abaixamentos manuais de carga (LAMC), uma contração isométrica voluntária máxima (CIVM inicial). Após um período de 10 minutos (evitando assim qualquer influência de fadiga), realizaram o LAMC seguido imediatamente por uma nova contração isométrica voluntária máxima (CIVM final) com o intuito de se avaliar a influência do LAMC sobre a CIVM. Ainda, após repouso de 10 minutos, realizaram novamente o mesmo protocolo (novo LAMC seguido de nova CIVM final)(Figura 1).

Para a manutenção da postura durante o teste, os voluntários receberam retorno visual por meio de um monitor conectado a uma câmera posicionada perpendicularmente a 4,96 metros do voluntário e 
1 metro do solo no plano sagital dos indivíduos. $\mathrm{O}$ ritmo do movimento foi padronizado em $40 \mathrm{bpm}$ por meio de um metrônomo digital.

A carga a ser elevada a partir do solo foi constituída de uma caixa de material plástico com $30 \mathrm{~cm}$ de altura, 35,5 cm de largura, 54,5 de comprimento, e uma perfuração na face lateral servindo como puxador. Dentro desta caixa foram depositadas anilhas, somando, aproximadamente, $15 \%$ e $25 \%$ do peso corporal de cada indivíduo.

O levantamento da carga foi realizado a partir da posição de joelho fletido e tronco ereto e o cinto pélvico inflável utilizado (Ehob-Air-Aligns ${ }^{\circledR}$ ) foi colocado ao nível da cintura dos voluntários.

As CIVMs (duração de cinco segundos) foram realizadas em um equipamento (Figura 2) disposto de uma célula de carga com limite de $100 \mathrm{Kg}$ (Kratos $\left.^{\circledR}\right)$. O músculo reto da coxa não foi avaliado isométricamente após os levantamentos e abaixamentos manuais de carga, pois o tempo despendido entre eles e as contrações isométricas de extensão de joelho, poderiam influenciar os registros EMG, não traduzindo a real influência do LAMC sobre esse músculo.

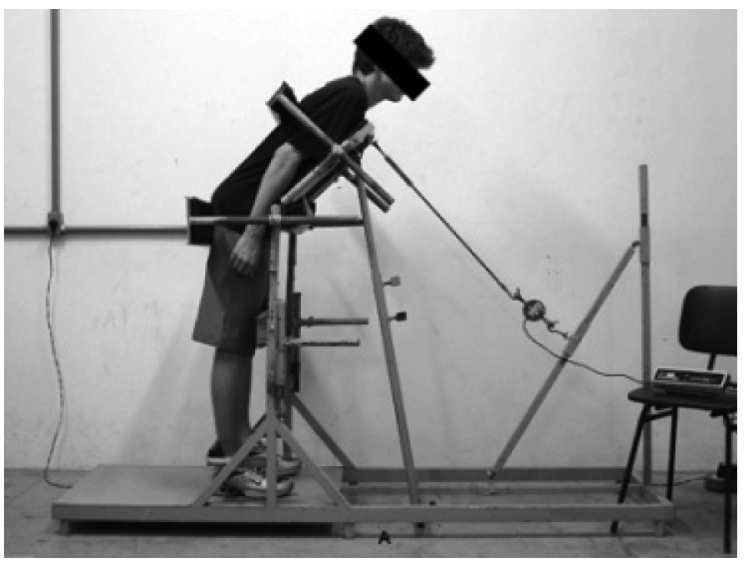

Figura 2. Equipamento MA ISOSTATION 2001

\section{EMG}

Para captação dos registros eletromiográficos, foram utilizados eletrodos de superfície (LECTEC), com revestimento de cloreto de prata com $2,8 \mathrm{~cm}$ de largura, $3,7 \mathrm{~cm}$ de comprimento, posicionados sobre o reto da coxa, segundo adaptação de Delagi et al. ${ }^{15}$ (metade da distância entre espinha ilíaca antero-superior e a borda superior da patela) e sobre o músculo iliocostal lombar, posicionados $10 \mathrm{~cm}$ acima da articulação sacro-ilíaca e $10 \mathrm{~cm}$ à direita, todos os músculos do lado direito. Para diminuir possíveis interferências na passagem do registro EMG, realizou-se anteriormente a colocação dos eletrodos uma tricotomia e limpeza da pele com álcool ao nível dos músculos estudados. Os voluntários foram também devidamente conectados a um fio-terra posicionado no punho direito.

Foi utilizado um módulo de aquisição de sinais biológicos $\left(\operatorname{Lynx}^{\circledR}\right)$ configurado com o ganho em 1000 vezes, filtro de passa-alta em $20 \mathrm{~Hz}$ e o filtro de passa-baixa em $500 \mathrm{~Hz}$. A conversão dos sinais analógicos para digitais foi realizada por uma placa A/D com faixa de entrada de -5 á +5 Volt (CAD 1026-Lynx $\left.{ }^{\circledR}\right)$. Para a aquisição dos sinais, foi utilizado um software específico (Aqdados-Lynx ${ }^{\circledR}$ ), configurado para uma freqüência de amostragem de $1000 \mathrm{~Hz}$. Para evitar a interferência da rede local no registro EMG, foi utilizado um filtro notch de $60 \mathrm{~Hz}$. Para existir um sincronismo entre a aquisição do registro eletromiográfico com o registro cinematográfico, foi utilizado um sistema foto eletrônico ${ }^{16}$. O sinal EMG foi coletado durante as contrações isométricas de 5 segundos e durante a atividade de levantamento e abaixamento manual de carga durante 1 minuto.

\section{Tratamento dos dados}

As variáveis EMG foram obtidas utilizando-se os procedimentos acima descritos em dois diferentes momentos: durante as contrações isométricas e durante os quatro protocolos de levantamento e abaixamento manual de carga.

Durante as ações isométricas foram obtidos os valores de Força de Tração na célula de carga $(\mathrm{Kg})$, Root Mean Square Médio $(\mathrm{RMSmed}=\mu \mathrm{V})$, Potencia Total (PT = unidade arbitraria), Freqüência média $(F M e d=\mathrm{Hz}$ ) e Freqüência mediana (FM $=\mathrm{Hz}$ ), obtidas do segundo de maior estabilidade, adquirido por meio de observação visual.

Durante o LAMC, após normalização do tempo, foram obtidos, a cada $2 \%$ de cada ciclo, os valores de RMS (normalizados pela CIVM, pela média e pico de atividade EMG), dos músculos reto da coxa e eretor da espinha.

\section{Método Estatístico}

Todos os valores aqui descritos estão expressos em média \pm desvio padrão e foi considerado o poder de $\alpha$ de 0,05 .

A influência dos diferentes dias de coleta sobre as variáveis obtidas durante a CIVM inicial (dia 1 $\mathrm{x}$ dia 2), bem como a influência do LAMC sobre as variáveis obtidas durante a CIVM (CIVM inicial x CIVM final) foi avaliada por meio do teste para duas variáveis dependentes de Wilcoxon.

Com o objetivo de identificar a reprodutibilidade da atividade eletromiográfica dos músculos 
eretor da espinha e reto da coxa, sobre os registros obtidos em todas as coletas durante a atividade de LAMC, determinou-se o Coeficiente de Variação inter-sujeitos para cada situação de teste (com cinto, com carga de 15\% peso corporal - CC15; sem cinto, com carga de $15 \%$ peso corporal - SC15; com cinto com 25\% peso corporal - CC25; sem cinto com carga de $25 \%$ peso corporal - SC25).

\section{RESULTADOS}

As cargas utilizadas foram de 9,38 $\pm 0,74 \mathrm{Kg}$ para o levantamento de $15 \%$ do peso corporal e de $15,38 \pm 0,74$ para o levantamento de $25 \%$ do peso corporal. Os voluntários realizaram em média 10,25 $\pm 1,16$ e 11,25 $\pm 0,89$ repetições no levantamento de $15 \%$ do peso corporal sem o cinto e com o

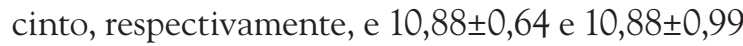
repetições no levantamento de $25 \%$ do peso corporal com o cinto e sem o cinto, respectivamente.

\section{CIVM inicial e final}

Uma vez que não houve diferenças estatisticamente significantes em nenhuma das variáveis entre as CIVM inicial do dia 1 e do dia 2, foi estabelecida a CIVM inicial do primeiro dia (para que houvesse uma condição de referência) para comparação entre o estado pré e pós a atividade de LAMC (Tabela 1).

As forças de extensão isométricas do tronco (Figura 3) apresentaram-se controversas após a atividade isotônica. Na situação CC15 e SC15,

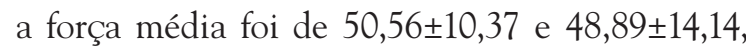
respectivamente e na situação CC25 e SC25 foi de $48,19 \pm 16,05,50,42 \pm 15,29$, respectivamente. Dentre estas situações com e sem o uso de cinto e com duas intensidades de contrações diferentes, houve apenas uma diminuição significativa na força de tração na célula de carga após o LAMC na situação CC25 ( $\mathrm{p}=0,035)$.

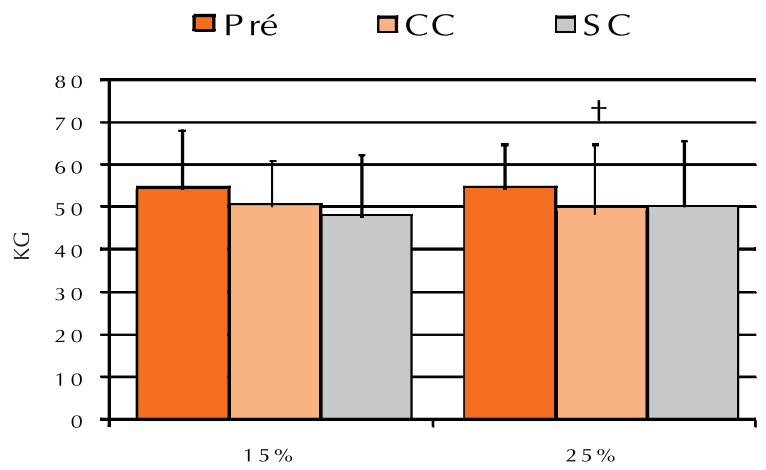

Figura 3. Valores de força de tração isométrica da célula de carga durante a extensão do tronco Pré e imediatamente após o teste de LMC repetitivo de $15 \%$ e $25 \%$ do peso corporal por um minuto com (CC) e sem (SC) cinto.

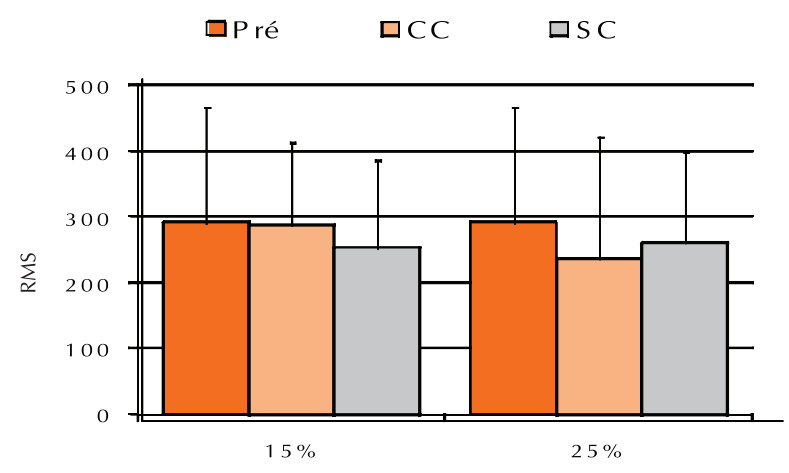

Figura 4. Valores de RMS médio $(\mu \mathrm{V})$ Pré e imediatamente após o teste de $\mathrm{LMC}$ repetitivo de $15 \%$ e 25\% do peso corporal durante um minuto com (CC) e sem (SC) cinto.

\section{EMG - Contrações isométricas}

Quanto ao comportamento da amplitude do sinal EMG do músculo eretor da espinha, a RMSMed (Figura 3) apresentou-se diminuída em todas as contrações isométricas após a realização do teste de LAMC, sendo que na situação SC15 houve um decréscimo mais evidente. Entretanto, a atividade EMG não apresentou diferença significativa entre as contrações realizadas antes e após o teste de LAMC, com ou sem cinto ( $p>0,05)$.

Ao se analisar as variáveis espectrais FMed e FM (Figuras 5 e 6) após o LMC sem a utilização do

Tabela 1. Valores da força de tração da célula de carga, RMS média, freqüência mediana, freqüência média e potência total durante a CIVM inicial realizadas no primeiro e segundo dias de coleta.

\begin{tabular}{lcc}
\hline \multirow{2}{*}{ Variável } & \multicolumn{2}{c}{ Contração inicial } \\
\cline { 2 - 3 } & Dia 1 & Dia 2 \\
\hline Cel $(\mathrm{Kg})$ & $54,55 \pm 13,61$ & $51,69 \pm 14,86$ \\
RMSMed $(\mu \mathrm{V})$ & $291,86 \pm 174,78$ & $272,70 \pm 183,73$ \\
FMed $(\mathrm{Hz})$ & $48,34 \pm 15,09$ & $50,30 \pm 24,48$ \\
FM (Hz) & $57,36 \pm 20,35$ & $64,73 \pm 29,40$ \\
PT (u.a.) & $29935,52 \pm 33024,02$ & $26632,34 \pm 32441,66$ \\
\hline
\end{tabular}

Cel - célula de carga; RMSMed - Root Mean Square Média; FM - Frequência mediana; Fmed - Frequência média; PT - Potência Total: u.a - unidade arbitraria. 
Tabela 2. Coeficientes de variação (CV) intersujeitos dos músculos eretor da espinha (ER) e reto da coxa (RC) quando normalizados pela contração voluntária máxima (CIVM), atividade média (MÉDIA) e pelo pico máximo de atividade (PICO), durante a primeira e oitava repetição do levantamento (L) e abaixamento (A) de carga de aproximadamente $15 \%$ e $25 \%$ do peso corporal, com (CC) e sem (SC) o uso de cinto pélvico.

\begin{tabular}{|c|c|c|c|c|c|c|c|c|c|c|c|c|c|c|}
\hline \multirow{3}{*}{$\stackrel{\stackrel{ }{\leftrightarrows}}{:}$} & \multirow{3}{*}{ ○ீ $\frac{\pi}{\tilde{\omega}}$} & \multirow{3}{*}{$\underset{\mathscr{\varpi}}{\mathscr{\varpi}}$} & \multicolumn{6}{|c|}{ Primeira repetição } & \multicolumn{6}{|c|}{ Oitava repetição } \\
\hline & & & \multicolumn{2}{|c|}{ CVM } & \multicolumn{2}{|c|}{ MÉDIA } & \multicolumn{2}{|c|}{ PICO } & \multicolumn{2}{|c|}{ CVM } & \multicolumn{2}{|c|}{ MÉDIA } & \multicolumn{2}{|c|}{ PICO } \\
\hline & & & ER & $\mathrm{RC}$ & ER & $\mathrm{RC}$ & ER & $\mathrm{RC}$ & ER & $\mathrm{RC}$ & ER & $\mathrm{RC}$ & ER & $\mathrm{RC}$ \\
\hline \multirow{3}{*}{$\mathrm{CC}$} & 15 & A & 61,4 & 72,1 & 33,3 & 34,5 & 28,7 & 36,4 & 62,0 & 87,3 & 33,3 & 31,9 & 27,8 & 31,4 \\
\hline & \multirow{2}{*}{25} & A & 54,0 & 62,4 & 40,0 & 21,2 & 30,3 & 21,7 & 51,6 & 65,9 & 35,6 & 27,1 & 32,0 & 26,1 \\
\hline & & $\mathrm{L}$ & 52,2 & 71,2 & 29,4 & 158,6 & 27,2 & 62,4 & 51,5 & 86,0 & 31,0 & 51,0 & 29,1 & 41,2 \\
\hline \multirow{2}{*}{ SC } & \multirow{2}{*}{15} & A & 65,3 & 77,5 & 25,3 & 20,8 & 26,0 & 23,7 & 60,9 & 87,5 & 40,4 & 26,5 & 30,7 & 27,9 \\
\hline & & $\mathrm{L}$ & 55,0 & 90,3 & 21,5 & 132,8 & 22,6 & 68,5 & 65,1 & 81,5 & 30,3 & 42,4 & 29,5 & 40,2 \\
\hline
\end{tabular}

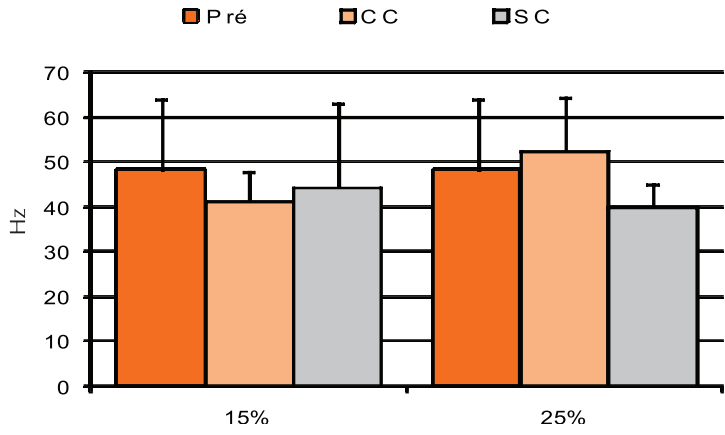

Figura 5. Valores de Fmed $(\mathrm{Hz})$ Pré e imediatamente após o teste de LMC repetitivo de $15 \%$ e $25 \%$ do PC por um minuto com (CC) e sem (SC) o uso do cinto pélvico.

口pré

口C $\quad$ a C

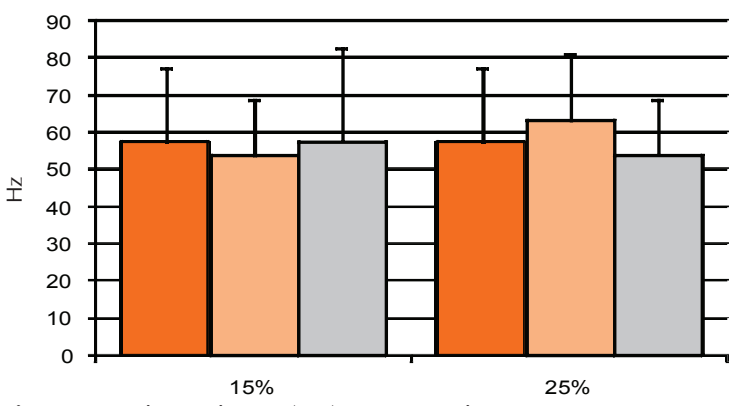

Figura 6. Valores de FM $(\mathrm{Hz})$ Pré e imediatamente após o teste de $\mathrm{LMC}$ repetitivo de $15 \%$ e $25 \%$ do $\mathrm{PC}$ por um minuto com (CC) e sem (SC) o uso do cinto pélvico.

cinto, foi encontrado um comportamento diferente frente à imposição de duas diferentes intensidades de carga (figura 5). Após o teste de levantamento de uma carga de $15 \%$ do peso corporal a FMed apresentou uma discreta diminuição com e sem a utilização de cinto. Já após o teste de levantamento de uma carga, correspondendo a $25 \%$ do peso corporal, as variáveis FM e Fmed apresentaram um comportamento diferente entre as situações CC e

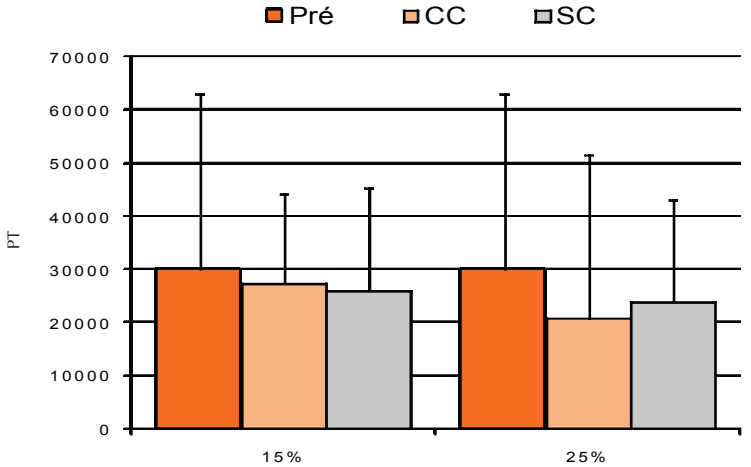

Figura 7. Valores de PT Pré e imediatamente após o teste de LAMC repetitivo de $15 \%$ e $25 \%$ do PC com (CC) e sem (SC) o uso de cinto pélvico.

SC, sendo que CC houve um aumento em relação à CIVM inicial e SC houve a diminuição. Entretanto em nenhuma das situações houve diferença significativa frente ao valor inicial $(\mathrm{p}>0,05)$.

Quanto aos valores de PT, estes se apresentaram diminuídos (em relação ao valor obtido durante a CIVM inicial, embora não apresentando diferenças significativas ( $\mathrm{p}>0.05)$ (Figura 7).

\section{Normalização e coeficiente de variação}

A tabela 2 mostra os valores de CV intersujeitos dos músculos eretores da espinha e reto da coxa, durante a primeira repetição de LAMC, correspondente a $15 \%$ e $25 \%$ do peso corporal, com e sem a utilização do cinto, normalizados pela CIVM, pela atividade média e pelo pico máximo de atividade desses mesmos músculos. Foram demonstradas a primeira e oitava repetições por serem situações extremas, entretanto, as demais repetições de forma geral foram semelhantes a essas. 
Os resultados demonstram que o CV apresentou menores valores quando obtido pelo pico, sendo os obtidos pela CIVM os que tiveram valores mais altos. Também nota-se que não há uma tendência predominante de aumento ou diminuição da primeira para a oitava série. Esses resultados se repetiram para diferentes situações como o uso e não uso do cinto pélvico, levantamento de $15 \%$ e $25 \%$ do peso corporal ou durante o levantamento ou abaixamento da carga.

\section{DISCUSSÃO}

A eficácia da utilização de cintos pélvicos na proteção da coluna lombar durante o levantamento manual de carga ainda apresenta discordância entre os diferentes estudos, embora, recentemente, PradoLeon et al..$^{17}$ ao estudar a forte correlação entre o LAMC com o acometimento de dores lombares, sugerem que $83 \%$ destas lombalgias poderiam ser prevenidas com a utilização de cintos pélvicos.

Desta forma, o fato desta atividade resistida ser realizada repetidamente como em algumas atividades de trabalho, um fator que deve ser considerado é o desenvolvimento da fadiga muscular, definida como a queda do desempenho ou a incapacidade de um músculo em manter uma força ou uma determinada carga de trabalho ${ }^{8}$. No presente estudo, esta incapacidade foi discretamente evidenciada por uma tendência à diminuição da força extensora após a utilização de ambas as cargas, com e sem a utilização do cinto, quando comparadas às condições pré LAMC. Entretanto, a única atividade capaz de produzir uma diminuição significativa de força foi quando se utilizou o cinto no LAMC correspondente a $25 \%$ do peso corporal. Sugerese que, ao elevar essa carga, o uso de cinto tenha promovido uma restrição no movimento da coluna lombar, capaz de provocar um maior esforço da musculatura da região para vencer essa restrição ${ }^{18}$. Outros autores concordam com essa idéia e relatam que grande parte dos indivíduos que utilizaram cintos por longos ou curtos ${ }^{19}$ períodos, relataram maior desconforto ao realizarem LAMC com o cinto pélvico.

$\mathrm{O}$ aumento da intensidade da carga durante o LAMC tem sido relacionado ao aumento das forças compressivas, da força de deslocamento vertebral e da atividade mioelétrica ${ }^{20}$, possibilitando, assim, nas intensidades de cargas mais altas, desencadearem lesões. Entretanto, de acordo com os resultados obtidos durante as contrações isométricas realizadas após o LAMC repetitivo, durante um minuto, com uma carga correspondente a $15 \%$ e $25 \%$ do peso corporal, não foram encontradas diferenças significativas na atividade muscular, o que sugere não ter sido uma intensidade de carga suficiente para promover uma fadiga evidente pelo período de tempo do teste.

Apesar de haver certa controversa, com alguns autores relatando sua diminuição ou manutenção ${ }^{21}$, normalmente, o comportamento da amplitude do sinal eletromiográfico em função do tempo, após uma tarefa, seja de um aumento pelo desenvolvimento da fadiga, devido ao recrutamento de novas unidades motoras para manter a tensão muscular ${ }^{22,23,24}$. Entretanto, no presente estudo, este aumento não foi evidente quando se realizou a CIVM após o LAMC repetitivo em qualquer das situações do estudo.

Um importante fator que influenciou os resultados encontrados foi o tempo de duração da atividade. Embora o LAMC possa ser realizado por diferentes freqüências de repetições e por tempos diferentes, a sua realização durante um minuto pode não ter sido suficiente para modificar os parâmetros eletromiográficos. Esse comportamento é contrário ao evidenciado por Bonato ${ }^{25}$, que ao utilizar cargas de $15 \%$ peso corporal durante atividade de LAMC encontrou diferenças significativas em todas as variáveis eletromiográficas após o término da atividade.

Este comportamento EMG também tem fortes implicações com o tempo de execução da tarefa, uma vez que modificações na FMed e também no torque têm sido mais bem evidenciadas após 4,5 minutos (Bonato, et $\mathrm{al}^{26}$ ) ou 15 minutos de LAMC (Potvin e Norman 1993) ${ }^{27}$. Após realizar 100 levantamentos de uma carga de $10 \mathrm{~kg}$, realizados de uma forma livre, somando-se 12,1 minutos, Dolan e Adams ${ }^{28}$ encontraram diferença significativa entre a FMed pré e a FMed após atividade para o segmento de L3 e não para T10.

Já segundo Jorgensen ${ }^{29}$, o tempo necessário para a recuperação da musculatura é proporcional ao tempo de resistência da mesma. Embora, como em nosso estudo, a duração da atividade proposta tenha sido de 1 minuto, apesar de a força de tração na célula de carga tenha se encontrado discretamente diminuída em todas as situações (Figura 3), a ausência de diferenças significativas nas variáveis eletromiográficas podem ser resultado do tempo entre o último LAMC e a realização da CIVM final, logo que períodos inferiores há 30 segundos tenham sido relatados como suficientes para a recuperação dos músculos da coluna lombar ${ }^{30}$. Esse tempo des- 
pendido entre o término do LAMC e as contrações isométricas é uma das limitações desse estudo.

Quanto ao comportamento da PT durante levantamentos e abaixamentos manuais de carga, a literatura apresenta uma escassez de informações, entretanto, os resultados obtidos neste estudo concordam com aqueles obtidos por Dolan e Adams ${ }^{28}$, que analisaram o efeito de 100 levantamentos e abaixamentos manuais de carga, no espectro de frequência EMG nos níveis de L3 e T10 e não encontraram diferenças no PT entre o estado pré e pós LAMC.

Durante o LAMC, um fator importante na prevenção de lesões tem sido atribuído à postura do corpo diante da carga a ser elevada. Snook ${ }^{31}$ conclui que o LAMC com coluna ereta e joelhos fletidos é mais recomendado para o levantamento de pequenos objetos, em relação à postura de inclinação do tronco para frente, a qual apresentou uma grande correlação com o nível de mudança nas variáveis espectrais, assim como Schultz, et al..$^{32}$ que encontraram os maiores valores de RMS dos músculos posteriores quando o tronco estava flexionado. Portanto, a postura adotada por esse estudo pode ter promovido estabilização suficiente para o tronco, diminuindo o desenvolvimento da fadiga muscular no tempo em que o movimento foi realizado.

Com intuito de identificar uma provável padronização do sinal eletromiográfico que represente um comportamento reprodutível de um músculo durante um movimento específico, a normalização apresenta-se como uma ferramenta que permite assim comparar diferentes voluntários quando submetidos à mesma tarefa. Este procedimento tem sido utilizado em biomecânica por diversos autores $^{12}$, particularmente para avaliar a reprodutibilidade do sinal eletromiográfico.

Embora a contração voluntária máxima seja muito utilizada com objetivo de normalização ${ }^{8}$, no presente estudo, quando o sinal EMG foi normalizado pela CIVM, os valores dos coeficientes de variação intersujeitos foram os que apresentaram valores maiores, concordando, assim, com Allison et al. ${ }^{33}$. Estes resultados também concordam com Yang e Winter ${ }^{11}$ que sugerem que os valores de referência sejam aqueles das contrações dinâmicas em relação àqueles obtidos de contrações isométricas devido as primeiras produzirem menores coeficientes de variação.

Este comportamento geral dos coeficientes de variação indica que, dentro da estrutura matemática deste índice, que é uma relação entre o desvio padrão e a média, um grande desvio padrão esteja embutido nesta análise e que, provavelmente, esteja relacionado com as diversas variáveis que influenciam um sinal eletromiográfico como a colocação dos eletrodos, a espessura do tecido adiposo, temperatura da pele e características físicas de cada voluntário.

\section{CONCLUSÃO}

O LAMC repetitivo durante um minuto com uma carga correspondente a $15 \%$ e $25 \%$ do peso corporal, com ou sem o uso do cinto, apresentou inexpressiva diferença na atividade muscular isométrica e isotônica, o que sugere não ter sido a intensidade de carga e o tempo, suficientes para promover uma fadiga evidente nos músculos retofemoral e iliocostal. Este fato nos permite duas afirmações: i) o tempo utilizado na realização dessa tarefa com as cargas de $15 \%$ e $25 \%$ do peso corporal, com a postura de joelhos fletidos e coluna ereta é seguro quanto à prevenção, logo que foi insuficiente para o desenvolvimento de fadiga nos músculos estudados; ii) a indicação do uso de cinto pélvico como acessório preventivo da dor lombar de forma aguda apresenta-se não eficaz do ponto de vista da ação muscular, promovendo até mesmo uma maior variabilidade do sinal EMG.

Os diferentes métodos de normalização da atividade EMG, com intuito de melhor comparação entre os indivíduos apresentam diferentes resultados, com menor variabilidade quando obtido pelo pico e pela média do sinal eletromiográfico,sendo, portanto, mais indicados durante a atividade proposta pelo presente estudo.

\section{REFERÊNCIAS BIBLIOGRÁFICAS}

1. McGill SM, Hughson RL, Parks K. Changes in lumbar lordosis modify the role of the extensor muscles. Clin Biomech 2000;15:777-780.

2. Hagen KB, Harms-Ringdahl K, Hallén J. Influence of lifting technique on perceptual and cardiovascular responses to submaximal repetitive lifting. Eur J Appl Physiol 2004;68(6):477-482

3. Gonçalves M, Cerqueira EP. Levantamento manual de carga a partir do solo com e sem o uso de cinto pélvico e com diferentes posturas de tronco: um estudo eletromiográfico. Rev Bras Biomec 2000;1(1):49-54.

4. Hagins M, Lamberg EM. Natural breath control during lifting tasks: effect of load. Eur J Appl Physiol 2006;96(4):453-8.

5. Sparto PJ, Parnianpour M. Estimation of trunk muscle forces and spinal loads during fatiguing repetitive trunk exertions. Spine 1998;23(23):2563-73.

6. Ortengren R, Andersson GBJ. Electromyographic studies of trunk muscles with special reference to the functional anatomy of the lumbar spine. Spine 1977;2(44-52. 
7. Moritani T, Nagata A, Muro M. Electromyographic manifestations of muscular fatigue. Med Sci Sports Exerc 1982;14(3):198-202.

8. Pereira MP, Gonçalves M. Efeito do gênero sobre fatigabilidade da musculatura lombar. Motriz 2006;12(1):43-50.

9. Gonçalves M, Barbosa FSS. Analise de Parâmetros de força e resistência dos músculos eretores da espinha lombar durante a realização de exercício isométrico em diferentes níveis de esforço. Rev Bras Med Esporte 2005;11(2):109-14.

10. Moritani T, Muro M, Kijima A, Berry MJ. Intramuscular spike analysis during ramp force output and muscle fatigue. Electromyogr Clin Neurophysiol 1986;26(2):147-60.

11. Yang JF, Winter DA. Electromyographic amplitude normalization methods: improving their sensitivity as diagnostic tools in gait analysis. Arch Phys Med Rehabil 1984;65(9):517-21.

12. Winter DA, Yack HJ. EMG profiles during normal human walking: stride-to-stride and inter-subject variability. Electroencephalogr Clin Neurophysiol 1987;67(5):402-11.

13. Knutson LM, Soderberg GL, Ballantyne BT, Clarke WR. A study of various normalization procedures for within day electromyographic data. J Electromyogr Kinesiol 1994;4(1):47-59.

14. Barbosa FSS, Gonçalves M. Comparação de Protocolos eletromiográficos utilizados para a identificação de sobrecarga na coluna lombar. Braz J Biomech 2005;6(10):27-33.

15. Delagi EF, Iazzetti J, Perotto A, Morrison D. Anatomic guide for electromyographer. The limbs. Ed. Charles C. Thomas; 1981.

16. Tortoza C, Gonçalves M. Montagem de um sistema de sinalização para aquisição simultânea de sinais eletromiográficos e cinematográficos. I Simpósio de Iniciação Cientifíca e V Encontro Interno de Trabalhos Cientifícos da Unesp promovido pel PET/ CAPES- Ciências Biológicas. 1993,

17. Prado-Leon LR, Celis A, Avila-Chaurand R. Occupational lifting tasks as a risk factor in low back pain: a case-control study in a Mexican population. Work Reading 2005;25(2):107-14.

18. Lavander SA, Tsuang YH, Hafez A, Andersson GBJ, Chaffin DB, Hughes RE. Coactivation on the trunk muscles during asymetric loading of the torso. Hum Factors 1992;34:239-47.

19. Reddell CR, Congleton JJ, Dale HR, Montgomery JF. An evaluation of a weightlifting belt and back injury prevention training class for airline baggage handlers. Appl Ergon 1992;23(5): 319-29.

20. Hall SJ. Effect of attempted lifting speed on forces and torque exerted on the lumbar spine. Med Sci Sports Exerc 1985;17(4):440-4.

21. Kumar S, Fagarasanu M, Narayan Y, Prasad N. Measures of localized spinal muscle fatigue. Egonomics 2006;49(11):1092-1110.

22. Arnall FA, Koumantakis GA, Oldham JA, Cooper RG. Between-days reliability of electromyographic measures of paraspinal muscle fatigue at 40, 50 and $60 \%$ levels of maximal voluntary contractile force. Clin Rehabil 2002;16:761-771.

23. Pereira MP, Gonçalves M, Barbosa FSS. Otimização de um protocolo para obtenção do limiar de fadiga eletromiográfico de músculos extensores lombares. Terapia Manual 2007;4:81-92.

24. Oliveira A, Grecco CC, Pereira MP, Gonçalves M, Denadai BS, Figueira TR, et al. Acute responses during a high resistance training session. J Strength Cond Research in press.

25. Potvin JR, Norman RW. Quantification of erector spinae muscle fatigue during prolonged, dynamic lifting tasks. Eur J Appl Physiol Occup Physiol 1993;67(6):554-62.

26. Bonato P, Boissy P, Della Cu, Roy SH. Changes in the surface EMG signal and the biomechanics of motion during a repetitive lifting task. IEEE Trans Neural Syst Rehabil Eng 2002;10(1):38-47.

27. Bonato, P Ebenbichler GR, Roy SH, Lehr S, Posch M, Kollmitzer J, et al. Muscle fatigue and fatigue-related biomechanical changes during a cyclic lifting task. Spine 2003;28(16):1810-20.

28. Dolan P, Adams MA. Repetitive lifting tasks fatigue the back muscles and increase the bending moment acting on the lumbar spine. J Biomech 1998;31(8):713-21.

29. Jorgensen K. Back muscle strength and body weight as limiting factors for work in the standing slightly-stooped position. Scand J Rehabil Med 1970; 2(149-53.

30. Snook SH. Comparison of different approaches for the prevention of low back pain. J Appl Ind Hygiene 1988;3:73-85.

31. Pereira MP, Gonçalves M. Extensão isométrica do tronco: análise da recuperação de parâmetros eletromiográficos. Fisiot Movim 2007;20(4):91-99.

32. Schultz A, Andersson GB, Ortengren R, Bjork R, Nordin M. Analysis and quantitative myoelectric measurements of loads on the lumbar spine when holding weights in standing postures. Spine 1982;7(4):390-7.

33. Allison DB. Limitations of coefficient of variation as index of measurement reliability. Nutrition 1993;9(6):559-61.

\footnotetext{
Endereço para correspondência

Mauro Gonçalves

Universidade Estadual Paulista Júlio

de Mesquita Filho.

Instituto de Biociências. Departamento

de Educação Física.

Programa de Pós-graduação em Ciências da Motricidade Humana. Campus Rio Claro, SP.

Av. 24A, 1515 - Bela Vista

CEP: 13506-900 - Rio Claro, SP

E-mail: maurog@rc.unesp.br
} 